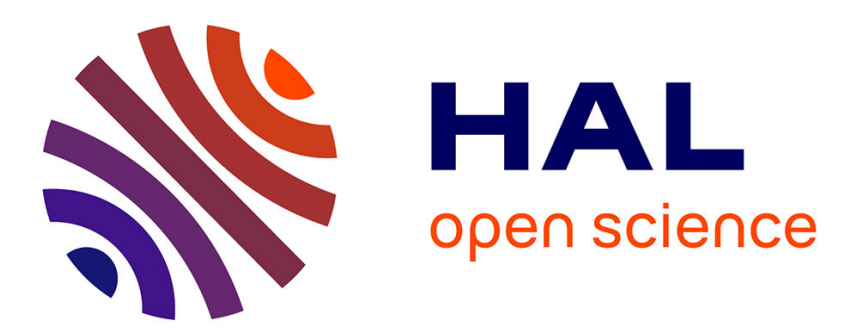

\title{
Une référence à la médecine de Cnide dans le débat philosophique entre Platon et Eudoxe
}

Victor Gysembergh

\section{To cite this version:}

Victor Gysembergh. Une référence à la médecine de Cnide dans le débat philosophique entre Platon et Eudoxe. Revue des Etudes Grecques, 2013, 126 (2), pp.615-622. 10.3406/reg.2013.8146 . hal02893668

\section{HAL Id: hal-02893668 \\ https://hal.univ-reims.fr/hal-02893668}

Submitted on 19 Mar 2021

HAL is a multi-disciplinary open access archive for the deposit and dissemination of scientific research documents, whether they are published or not. The documents may come from teaching and research institutions in France or abroad, or from public or private research centers.
L'archive ouverte pluridisciplinaire HAL, est destinée au dépôt et à la diffusion de documents scientifiques de niveau recherche, publiés ou non, émanant des établissements d'enseignement et de recherche français ou étrangers, des laboratoires publics ou privés. 


\section{Victor GYSEMBERGH}

\section{UNE RÉFÉRENCE À LA MÉDECINE DE CNIDE DANS LE DÉBAT PHILOSOPHIQUE ENTRE PLATON ET EUDOXE*}

Eudoxe de Cnide, le célèbre savant du quatrième s. av. n. è. ${ }^{1}$, paraît être un témoin fort bien placé pour livrer des renseignements sur l'école médicale de Cnide telle qu'elle se présentait à son époque. Il n'est en effet pas seulement un des plus grands mathématiciens et astronomes de son temps, un savant dont les recherches influencèrent notamment Platon et Aristote. Diogène Laërce, s'appuyant sur Sotion, le qualifie aussi de médecin et nomme, parmi ses fréquentations, un certain Théomédon, médecin, avec lequel il aurait effectué un voyage de jeunesse à Athènes. On peut supposer que Théomédon était aussi Cnidien, parce qu'il était suffisamment proche d'Eudoxe, qui était pauvre, pour accepter de subvenir à ses besoins (D.L. VIII, 86). Eudoxe aurait aussi connu le médecin Chrysippe de Cnide, fils de Crinès, avec lequel il se rendit en Égypte (D.L. VIII, 87). Eudoxe et Chrysippe furent tous deux élèves de Philistion de Locres (D.L. VIII, 86 et 89), et le second suivit l'enseignement du premier sur certaines matières étrangères à la médecine. Lors de leur voyage en Égypte, ils bénéficièrent de

* Qu'il me soit permis de remercier ici MM. Jacques Jouanna, Didier Marcotte et Antoine Pietrobelli pour leur relecture et leurs conseils. Je demeure seul responsable de toutes les imperfections.

1 Sur les dates d'Eudoxe cf. en dernier lieu H.-J. Waschkies, Von Eudoxos zu Aristoteles : Das Fortwirken der Eudoxischen Proportionentheorie in der Aristotelischen Lehre vom Kontinuum, Amsterdam, 1977, p. 34-58. 
lettres d'introduction d'Agésilas, roi de Sparte (D.L. VIII, 87), cité qui était la métropole de Cnide ; ces lettres leur permirent d'étudier auprès de prêtres égyptiens ${ }^{2}$

Ces données, qui montrent qu'Eudoxe était bien introduit dans le milieu médical cnidien et suggèrent que son exceptionnelle culture scientifique incluait aussi la médecine, nous invitent à prendre au sérieux une indication de doxographie médicale qui figurait dans son traité de géographie intitulé Circuit de la Terre $^{3}$. Cette indication nous est transmise par Diogène Laërce (VIII, $90=$ Eudoxe de Cnide, F 339 Lasserre $^{4}$ ) :

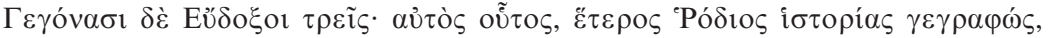

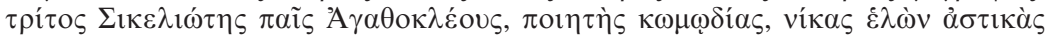

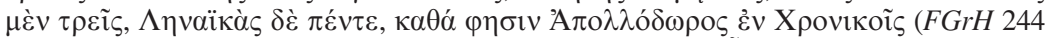

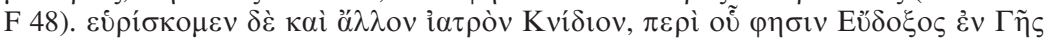

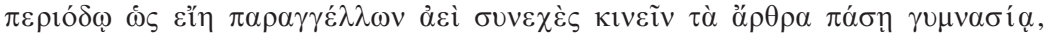

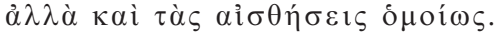

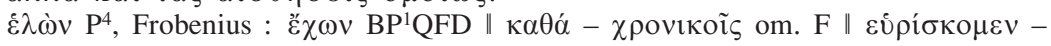
ó ó́ $\omega \varsigma$ ad testimonium de Chrysippo (D.L. VIII, 89) transp. Wilamowitz (Antigonos

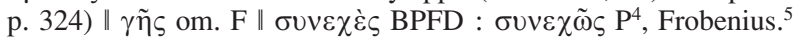

« h y eut trois Eudoxe : celui-là même ( $s c$. Eudoxe de Cnide), un autre de Rhodes qui écrivit des Histoires, un troisième, Sicilien, fils d'Agathoclès, poète de comédie, qui remporta trois victoires au concours de sa ville et cinq aux Lénéennes, comme le dit Apollodore dans les Chroniques. Nous en trouvons encore un autre, médecin de Cnide, dont Eudoxe dit dans son Circuit de la Terre qu'il prescrivait toujours de constamment mouvoir les articulations par toute sorte d'exercice, mais aussi les sensations, de la même manière. »

Ce passage semble avoir été peu pris en compte par les historiens de la médecine, malgré son importance, notamment, pour l'histoire de l'école médicale de Cnide $^{6}$. Ainsi, il ne figure pas dans l'étude des plus anciens témoignages relatifs à l'école de Cnide par H. Grensemann ${ }^{7}$. Ce paradoxe trouve peut-être son explication dans une querelle virulente qui survint au début du Xx ${ }^{\text {ème }}$ siècle. En effet, l'école médicale de Cnide a compté, au Iv ${ }^{\text {ème }}$ s. av. n. è., plusieurs médecins portant

2 Cnide faisait aussi partie des cités grecques ayant reçu du pharaon Amasis le privilège de commercer librement en Égypte, cf. Hérodote, Histoires II, 178.

3 Sur le titre Circuit de la terre $(\gamma \tilde{\eta} \varsigma \pi \varepsilon \rho \operatorname{ó} \delta \mathrm{o} \varsigma)$, que portait déjà le traité géographique composé par Hécatée de Milet, cf. D. Marcotte, Les géographes grecs. I. Introduction générale. Pseudo-Scymnos, Paris, 2000, p. LXI-LXII. Le Circuit de la terre d'Eudoxe, qui présentait une méthode novatrice permettant la construction mathématique de la carte terrestre, contenait aussi, conformément à la tradition du genre, d'abondants détails de géographie physique et humaine.

4 Cf. F. Lasserre, Die Fragmente des Eudoxos von Knidos, Berlin, 1966, p. 118.

5 Les apparats critiques sont composés par l'auteur à partir des éditions de H.S. Long, Diogenis Laertii Vitae Philosophorum, Oxford, 1964 ; M. Marcovich, Diogenis Laertii Vitae Philosophorum, Stuttgart-Leipzig, 1999 ; et T. Dorandi, Lives of Eminent Philosophers. Diogenes Laertius, Cambridge, 2013.

6 Sur l'existence d'une école de Cnide, qui fait encore débat aujourd'hui, cf. la synthèse récente de J. Jouanna, "Postface », dans la deuxième édition de Hippocrate. Pour une archéologie de l'école de Cnide, Paris, ${ }^{2} 2009$.

7 H. Grensemann, Knidische Medizin. I. Die Testimonien zur ältesten knidischen Lehre und Analysen knidischer Schriften im Corpus Hippocraticum, Stuttgart, 1975, p. 206. 


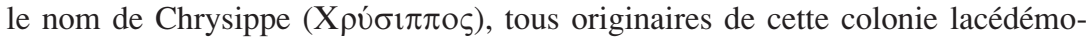
nienne située en Carie, sur la péninsule appelée Chersonèse dorienne. Ces homonymes nous sont connus par plusieurs témoignages que transmettent des auteurs postérieurs. L'interprétation de ces témoignages, et notamment la question du nombre d'individus différents auxquels ils se rapportent, a suscité une vive

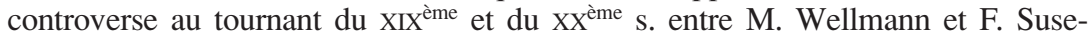
mihl ${ }^{8}$. Le problème des Chrysippe de Cnide n'a, depuis lors, toujours pas reçu de réponse qui fasse consensus ${ }^{9}$, et l'édition des témoignages concernant ces médecins n'a jamais été entreprise ${ }^{10}$.

Or depuis Wilamowitz, qui proposait de déplacer l'indication doxographique fournie par Eudoxe, on a parfois voulu rapporter celle-ci à un développement précédent, qui concerne les rapports d'Eudoxe et de Chrysippe de Cnide, ainsi que la carrière et la postérité de ce dernier (D.L. VIII, 89) ${ }^{11}$ :

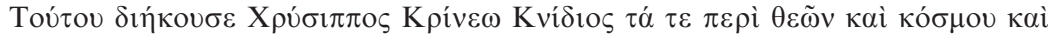

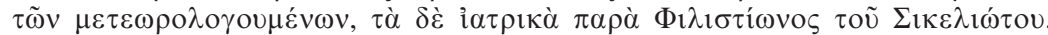

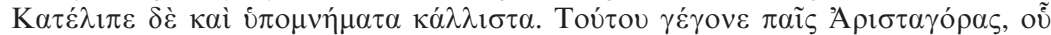

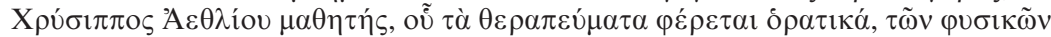

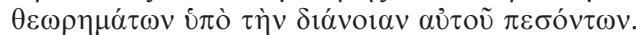

Kpíve心 Bergk (Kleine Schriften II, 288), Croenert (Kolotes, 189) coll. Suda $\Sigma 446$ :

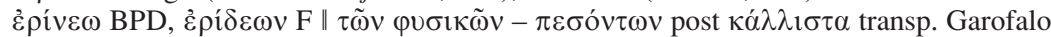
(Erasistrati Fragmenta p. 21)

"C'est lui (sc. Eudoxe) dont Chrysippe de Cnide, fils de Crinès, suivit l'enseignement à propos des dieux, de l'univers et des choses célestes, tandis qu'il suivit celui de Philistion le Sicilien pour la médecine. Il ( $s c$. Chrysippe) laissa de très beaux traités. Il eut pour fils Aristagoras, qui eut pour fils Chrysippe l'élève d'Aéthlios ; de ce Chrysippe sont transmis les Soins de la vue, parce que sa réflexion a porté sur les observations physiques. »

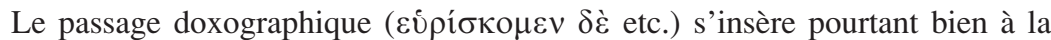
suite de la notice de Diogène concernant les homonymes d'Eudoxe. Comme le note J.-F. Balaudé, la place du passage doxographique dans le texte transmis, faisant immédiatement suite à la notice sur les homonymes d'Eudoxe de Cnide, laisse penser que le médecin cnidien dont il est question est un quatrième Eudoxe

8 Cf., par ordre chronologique : F. Susemihl, « Die Lebenszeit des Eudoxos von Knidos », $R h M$ 53, 1898, p. 626-628 ; M. Wellmann, « Chrysippos » 15, RE III 2, 1899, col. 25092510 ; id., «Zur Geschichte der Medicin im Alterthum », Hermes 35, 1900, p. 349-384 ; F. Susemihl, «Chrysippos von Knidos und Erasistratos », RhM 56, 1901, p. 313-318; M. Wellmann, « Chrysippos » 15 et 16, RE Suppl. I, 1903, col. 299 ; id., « Erasistratos » 2, $R E$ VI 1, 1907, col. 333-350.

9 Cf. en dernier lieu R. Goulet, «Chrysippe de Cnide », in : Dictionnaire des philosophes antiques, vol. II, Paris, 1994.

10 Une grande partie des témoignages est mentionnée par M. Wellmann, « Chrysippos » 15, RE III 2, 1899, col. 2509-2510. On ajoutera notamment un passage de l'Anonymus Bambergensis (Staatsbibliothek Bamberg, L.III.B (med. 1), fo $6^{r}=$ Herophilus fr. 16a von Staden) et le commentaire d'Eustathe de Thessalonique au vers $\Lambda 514$ de l'Iliade (III, p. 243, 40-42 van der Valk).

11 Cf. U. von Wilamowitz-Moellendorff, Antigonos von Karystos, Berlin, 1881, p. 324. 
plutôt qu'un personnage nommé Chrysippe ${ }^{12}$. L'intervention de Wilamowitz, qui déplace la phrase, n'est justifiée par aucun indice d'une perturbation textuelle. Ni H.S. Long, ni M. Marcovich, ni T. Dorandi n'acceptent cette intervention dans leurs éditions respectives ${ }^{13}$. Comme l'explique J. Mejer, Diogène Laërce place souvent, à la fin d'une biographie, une série d'excerpta ${ }^{14}$. Le passage doxographique extrait du Circuit de la Terre constitue donc l'ajout, signalé par Diogène à la première personne du pluriel, d'un quatrième Eudoxe qui ne se trouvait pas dans la liste d'homonymes fournie par les Chroniques d'Apollodore ; il ne saurait s'agir d'un des Chrysippe.

Il est vraisemblable que cet Eudoxe médecin, qu'on peut appeler, pour le distinguer de son célèbre homonyme et sans préjuger de leur chronologie respective, Eudoxe (de Cnide) alter, appartenait à la même famille que l'auteur du Circuit de la Terre. Ainsi, Ctésias de Cnide témoignait, dans un fragment d'origine inconnue, que l'ellébore n'était pas employée par les médecins du temps de son père et de son grand-père ${ }^{15}$. Au reste, il n'est que de songer à l'exemple des Hippocrate, Asclépiades de Cos, pour comprendre que c'était une pratique répandue, pour une dynastie de savants jouissant d'un certain renom, d'entretenir ce prestige en transmettant leur nom de génération en génération. On compte en effet au moins huit médecins portant ce nom, depuis Hippocrate I, le grand-père du célèbre Hippocrate II (dit le Grand), en passant par Hippocrate III et IV, les petits-fils d'Hippocrate II, jusqu'à Hippocrate VII, fils de Praxianax, lui aussi un Asclépiade de Cos, sans oublier Hippocrate V et VI, fils de Thymbraios, le disciple d'Hippocrate II, et enfin Hippocrate VIII, connu par une inscription d'époque hellénistique, et qui fut vraisemblablement un descendant d'Hippocrate II appartenant à la branche de Thessalos ${ }^{16}$.

Eudoxe de Cnide alter était-il apparenté à son homonyme, l'auteur du Circuit de la Terre ? Quoi qu'il en soit, ce médecin semble avoir été encore en vie au moment de la rédaction du Circuit de la Terre. En effet, le témoignage doxographique est rapporté par Diogène à l'optatif présent ( $\omega \zeta$ Eín $\pi \alpha \rho \alpha \gamma \gamma \varepsilon \dot{\varepsilon} \lambda \lambda \omega v)$. Or dans le discours indirect à l'optatif oblique, l'optatif présent ne peut représenter un imparfait du discours direct qu'à de rares occasions, uniquement si le contexte permet d'établir clairement que l'optatif représente un imparfait plutôt qu'un présent ${ }^{17}$. Ce n'est pas le cas ici : le texte du Circuit de la Terre était donc, semble-t-il, formulé au présent, ce qui ferait d'Eudoxe de Cnide alter à tout le moins, sinon un parent, du moins un contemporain d'Eudoxe de Cnide.

12 J.-F. Balaudé, in : Marie-Odile Goulet-Cazé (dir.), Diogène Laërce. Vies et doctrines des philosophes illustres, trad. fr., Paris, 1999, p. 1018, n. 7.

13 Cf. supra, n. 5.

14 J. Mejer, Diogenes Laertius and his Hellenistic Background, Wiesbaden, 1978, p. 34, n. 71 : «The two references to Apollodorus in 8.90 are separated by what looks like another excerpt, i.e. the mention of a fourth Eudoxus who evidently did not appear in the list of homonyms, and are followed by yet another excerpt from Favorinus. As often Diogenes collects a series of excerpts at the end of a biography. »

15 Cf. Ctésias de Cnide, F 68 Lenfant (= Oribase, Recueils médicaux 8.8).

16 Cf. J. Jouanna, Hippocrate, Paris, 1992, p. 69, p. 71 et p. 81-82.

17 Cf. W.W. Goodwin, Syntax of the Moods and Tenses of the Greek Verb, Londres, $1889^{2}, \S 673$. 
Dans cet extrait du Circuit de la Terre, il paraît s'agir d'une panacée, d'un remède universel. Celui-ci relève, plus précisément, de la diététique. Un parallèle frappant se trouve dans le Timée de Platon (écrit après 360 av. n. è.) : l'interlocuteur principal, Timée de Locres, un savant sicilien de la deuxième moitié du $\mathrm{v}^{\text {ème }} \mathrm{s}$. av. n. è., prône le mouvement conjoint du corps et de l'âme comme le seul remède aux deux espèces de maladies $(88 b-c)$ :

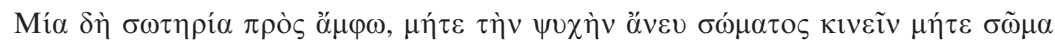

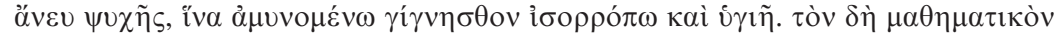

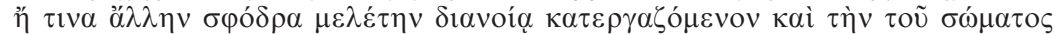

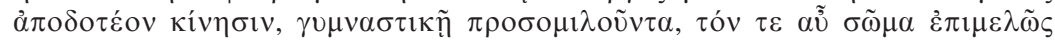

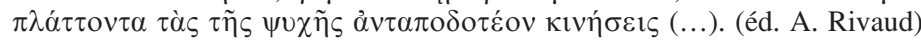

«Contre ces deux maladies il n'y a qu'un remède : ne mouvoir jamais l'âme sans le corps, ni le corps sans l'âme, afin que, se défendant l'un contre l'autre, ces deux parties gardent leur équilibre et leur santé. Il faut donc que le mathématicien et quiconque exerce énergiquement quelque activité intellectuelle, donne aussi du mouvement à son corps et qu'il pratique la gymnastique. Inversement celui qui cultive soigneusement son corps doit aussi donner à l'âme les mouvements compensateurs (...). » (trad. A. Rivaud)

Après un développement rattachant cette idée thérapeutique aux thèses cosmologiques dont il s'est fait l'auteur plus tôt dans le dialogue, Timée ajoute, comme une condition à l'efficacité de ce traitement, la nécessité du caractère continu du mouvement, qui doit contrecarrer le mouvement des substances entrant dans le corps et celui des objets qui l'affectent de l'extérieur (87d) :

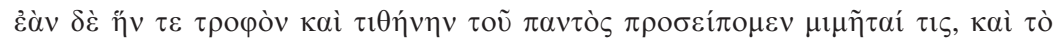

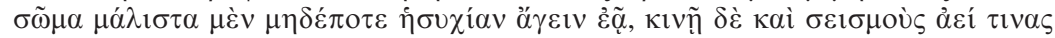

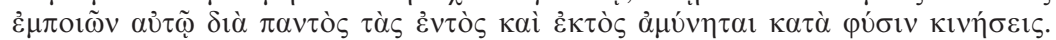
(éd. A. Rivaud)

« Si imitant cette nature que nous appelions la nourrice et la mère du Tout, on ne permet jamais au corps de demeurer au repos, si constamment on lui imprime quelque agitation, il saura toujours se défendre naturellement contre les mouvements intérieurs ou extérieurs. » (trad. A. Rivaud)

Enfin, Timée justifie la primauté de la gymnastique comme remède, parce qu'elle est issue de l'action propre du corps (89a) :

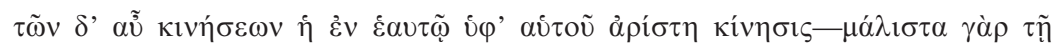

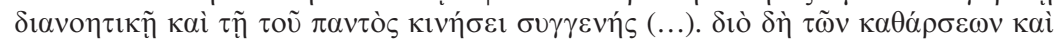

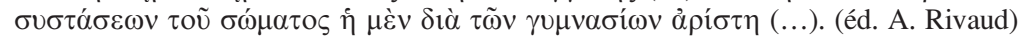

«Parmi les mouvements du corps, le meilleur est celui qui naît en lui par son action propre, car c'est le plus conforme au mouvement de l'Intelligence et à celui du Tout (...). Par suite, de tous les moyens de purifier et de disposer le corps, le meilleur est celui qui s'obtient par les exercices gymnastiques. » (trad. A. Rivaud)

Il est aisé de déceler dans ces passages de Platon des thèmes philosophiques sans doute étrangers à la thérapie proposée par Eudoxe alter, tels que l'analogie entre les arts permettant de soigner le corps et ceux qui permettent de soigner l'âme, bien connue d'autres dialogues platoniciens (par exemple Gorg., 464b 466a), ou encore la conception toute pythagoricienne de la santé comme un équilibre (cf. Alcméon de Crotone, 24 B 4 Diels-Kranz). Les extraits reproduits ci-dessus ne rendent qu'imparfaitement compte de l'art avec lequel Platon et son 
personnage Timée marient différentes traditions de pensée en entremêlant les kôla et les kommata en de longues périodes. Par comparaison, le propos doxographique d'Eudoxe, tel que rapporté par Diogène, confine au prosaïsme. Le contexte a beau être perdu, il est tentant d'y voir une allusion railleuse au dialogue de Platon. Eudoxe fréquenta Platon et l'Académie, et ces relations donnèrent lieu à une tradition faisant d'Eudoxe l'élève de Platon (Sotion d'Alexandrie apud D.L. VIII, 86). La Lettre XIII du corpus platonicien garde la trace d'échanges entre Platon et l'école d'Eudoxe, représentée en l'espèce par Hélicon de Cyzique. Par ailleurs, le Timée suscita dès sa divulgation d'intenses débats philosophiques au sein de l'Académie ${ }^{18}$.

Nous défendrons l'hypothèse suivante : dans le Circuit de la Terre, Eudoxe aurait allégué la doctrine de son homonyme pour souligner l'absence d'originalité de la thérapeutique platonicienne ; ce faisant, il invitait son lectorat à percevoir l'abîme séparant la prolifération d'arguments produite par le philosophe homme de lettres de la formule lapidaire sans cesse répétée par le praticien, homme d'expérience $^{19}$. À cet égard, il est intéressant de noter que Timée vient « d'une ville pourvue d'une excellente constitution, Locres en Italie », comme Philistion, le maître d'Eudoxe en médecine ${ }^{20}$. Les références à Eudoxe sont nombreuses dans les dialogues platoniciens : il tombait notamment sous le coup (peut-être parmi d'autres) du destructeur examen dialectique de l'hédonisme dans le Philèbe ${ }^{21}$.

Pour saisir la portée de la référence médicale dans le Circuit de la Terre, les textes d'Eudoxe et de Platon doivent encore être rapprochés d'un troisième

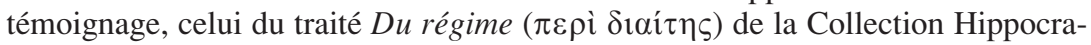
tique. Notons d'emblée que par plusieurs caractéristiques (schéma des descriptions nosologiques, importance et teneur de la diététique), ce traité se rapproche de la médecine cnidienne ${ }^{22}$; toutefois, les indices qu'on peut relever ne suffisent pas à établir définitivement une origine cnidienne. Peut-être plus importante est l'association particulière du traité avec l'astronomie d'Eudoxe de Cnide. En effet, il se singularise au sein du Corpus Hippocratique par l'indication d'ö $\sigma \tau \rho \omega v$ $\delta 1 \alpha \sigma \tau \eta ́ \mu \alpha \tau \alpha$, c'est-à-dire d'intervalles comptés en jours séparant divers événements astronomiques (phases, solstices et équinoxes) au cours de l'année ${ }^{23}$. Or ce passage correspond étroitement au calendrier astronomique d'Eudoxe ${ }^{24}$.

Le Régime présente en outre la particularité de donner une étude d'ensemble de la nature humaine - une anthropologie - à laquelle est consacré le premier livre dans son intégralité : en particulier, la doctrine de l'âme ou psychologie (I, 35-36) est semblable en bien des points à celle du Timée $e^{25}$. Or la psychologie

18 Sur la réception rapide du Timée après sa publication, cf. A. Taylor, A Commentary on Plato's Timaeus, Oxford, 1928, p. 1-2.

19 Je remercie vivement M. Marwan Rashed de m'avoir aidé à formuler la relation entre ce passage d'Eudoxe et Platon.

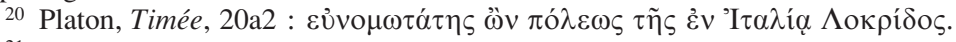

21 Cf. notamment Philèbe, $11 \mathrm{~b}$ et $67 \mathrm{~b}$; Timée, 39b-e ; Lois, 882a.

22 Cf. R. Joly, Hippocrate. Du régime (= CMG I 2, 4), Berlin, 1974, p. 38.

${ }^{23}$ Du régime, I.594-604, p. 194, 23 - 200, 22 Joly.

24 Cf. A. Rehm, s.v. «Parapegma », RE 18/4, col. 1328-9, et l'opinion contraire de R. Joly, op. cit., p. 48.

25 Cf. J. Jouanna, « La théorie de l'intelligence et de l'âme dans le traité hippocratique 'Du régime' : ses rapports avec Empédocle et le 'Timée' de Platon », REG 79, 1966, 
du traité Du régime est le seul passage de la Collection Hippocratique où il soit question non de la sensation ( $\alpha i \jmath \sigma \eta \eta \sigma \varsigma)$, mais, comme chez Eudoxe alter, des sensations ( $\alpha i \sigma \theta \eta \dot{\sigma} \varepsilon \varsigma)$. Cette théorie hippocratique des sensations est justement un des points qui se rapprochent le plus du Timée : le degré d'intelligence d'une âme $(\psi v \chi \eta ি)$ dépend de la rapidité avec laquelle celle-ci effectue sa révolution

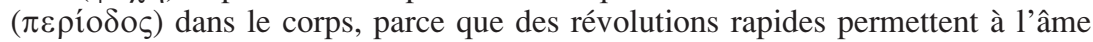

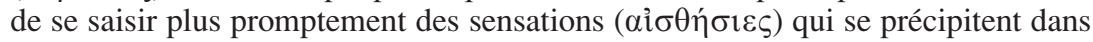
le corps par les canaux des sens (Du régime I.355, p. 152, 30-31 [Joly] $)^{26}$ :

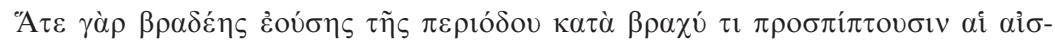

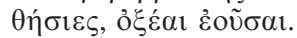

« Étant donné, en effet, que la révolution (de l'âme) est lente, les sensations ${ }^{27}$ n'ont qu'un court instant à chaque fois pour y arriver quand elles sont rapides. » (trad. R. Joly)

Comme l'a montré J. Jouanna, la théorie de l'intelligence développée dans le Régime est, à son tour, l'héritière de celle d'Empédocle (apud Théophraste, De sensu $10-11=31$ A 86 Diels-Kranz $)^{28}$. Toutefois, si pour le pythagoricien d'Agrigente, c'était la circulation du sang qui expliquait les différences de vivacité entre les âmes, il revient à l'auteur du Régime d'avoir introduit la révolution de l'âme comme principe explicatif de l'intelligence.

Retracer cette généalogie du texte platonicien, qui paraît remonter à Empédocle par l'intermédiaire, direct ou indirect, du traité hippocratique $D u$ régime, permet de mieux cerner l'enjeu proprement philosophique de l'opinion rapportée par Eudoxe dans son Circuit de la Terre. ll s'agit certes pour le Cnidien de diriger une pique railleuse contre Platon, et peut-être de lui rappeler sa dette envers l'école cnidienne. Néanmoins, la référence médicale permet aussi de souligner un présupposé laissé sans questionnement par Platon et ses prédécesseurs, à savoir l'impossibilité d'agir sur les sensations elles-mêmes. D'Empédocle au Timée, il n'est question d'agir que sur le mouvement interne de l'intelligence : en signalant la pensée de son homonyme, Eudoxe suggère que l'homme peut intervenir aussi sur l'autre paramètre de l'intéraction, c'est-à-dire sur les sensations (notamment en agissant sur les stimulus extérieurs dont elles découlent).

C'est donc le statut des sensations qui est implicitement visé, un problème philosophique à propos duquel Eudoxe et Platon s'opposaient par bien des aspects ${ }^{29}$. L'hédonisme d'Eudoxe, qui proclamait que le plaisir est le souverain bien parce

p. XV-XVIII ; comm. ad loc. de R. Joly, op. cit. ; J. Jouanna, « La théorie de la sensation, de la pensée et de l'âme dans le traité hippocratique du Régime : ses rapports avec Empédocle et le Timée de Platon », AION. Annali dell'università degli studi di Napoli "L'Orientale ». Dipartimenti di studi del mondo classico e del Mediterraneo antico. Sezione filologico-letteraria 29, 2007, p. 9-38.

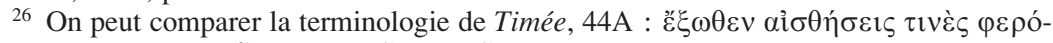

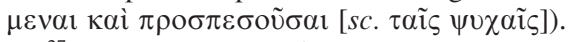

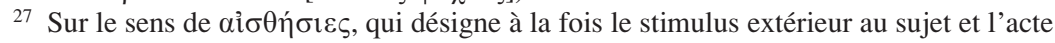
perceptif qui en découle, cf. J. Jouanna, «La théorie de la sensation, de la pensée et de 1'âme... », art. cit. (n. 25), p. 21-26.

28 J. Jouanna, « La théorie de la sensation, de la pensée et de l'âme... », art. cit. (n. 25), p. 26-31.

${ }^{29}$ La critique par Eudoxe de la théorie platonicienne des Idées, qui dépasse le cadre de cette étude, fera l'objet ultérieurement d'une enquête plus approfondie. 
que toutes choses tendent vers celui-ci (cf. Eudoxe de Cnide, D 3 Lasserre), n'était pas soluble dans le platonisme : il présupposait notamment d'accorder à la sensation une valeur épistémologique que Platon lui refusait au profit des réalités mathématiques et idéales. Eudoxe développait les conséquences de sa position philosophique par rapport à celle de Platon en examinant aussi la théorie platonicienne des idées, c'est-à-dire en étendant sa réflexion au champ de l'ontologie. Le contenu

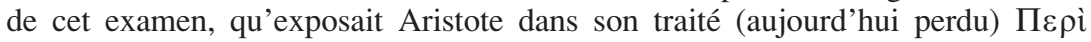
i $\delta \varepsilon \tilde{\omega} v$, est difficile à reconstituer, parce que le statut du témoignage d'Alexandre d'Aphrodise à ce sujet (= D 2 Lasserre) est peu clair ${ }^{30}$. Néanmoins le fait qu'il s'agissait d'une critique de la théorie platonicienne des idées est attesté explicitement par Aristote (Métaphyique M, 1079 b 18-22) :

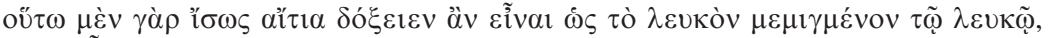

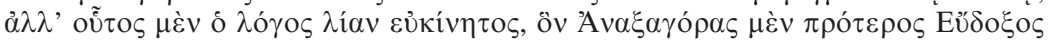

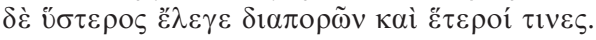

Peut-être sembleraient-elles ( $s c$. les idées) être des causes de même que le blanc mêlé au blanc, mais ce raisonnement est trop facile à réfuter, qu'a tenu en premier Anaxagore, ensuite Eudoxe, qui énumérait les difficultés ( $s c$. de la théorie des idées), et d'autres.

La précision $\delta 1 \alpha \pi \circ \rho \tilde{\omega} v$ ne figure pas dans la version parallèle qui se trouve en Métaphysique A, 991 a 14-18. Le caractère essentiellement critique des développements d'Eudoxe à propos de la théorie des idées a donc échappé le plus souvent à l'attention des commentateurs à cause d'un choix éditorial discutable de F. Lasserre, qui imprime la seule rédaction de Métaphysique A, sans rendre compte des divergences de la rédaction parallèle contenue dans le livre $\mathrm{M}^{31}$. Cette critique était couplée à une théorie du mélange ou de la participation des idées dans la réalité

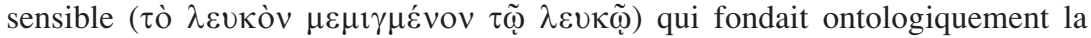
valeur morale et épistémologique de la sensation, et notamment du plaisir.

En somme, si l'importance des recherches scientifiques d'Eudoxe de Cnide pour la philosophie de Platon est généralement reconnue, il semble que le Cnidien ait aussi développé une critique proprement philosophique de son contemporain. Cette critique s'appuyait sur une connaissance approfondie des théories médicales de son temps. Tout en nous donnant une image vivante du débat intellectuel en Grèce au siècle de Platon et d'Aristote, l'étude de la référence faite par Eudoxe de Cnide à la diététique de son homonyme Eudoxe de Cnide alter atteste l'activité du premier dans les domaines de la médecine et de la philosophie, invitant le philologue à prendre la mesure de ce qui s'est perdu dans le cours des siècles, non pour qu'il renonce à ses recherches, mais pour qu'il s'y attache avec toute l'acribie nécessaire.

Victor GYSEMBERGH

(Université de Reims)

\footnotetext{
${ }^{30}$ Cf. en dernier lieu G. Fine, On Ideas : Aristotle's Criticism of Plato's Theory of Forms, Oxford, 1995 , c. 3 , n. 24 et 34.

31 Cf. F. Lasserre, op. cit., p. 12.
} 